\title{
Optimization in Fuzzy Inventory Model for Linearly Deteriorating Items, with Power Demand, Partial Backlogging and Linear Holding Cost
}

\author{
N. Rajeswari \\ Sri GVG Visalakshi College for \\ Women
}

\author{
T. Vanjikkodi \\ Sri GVG Visalakshi College for \\ Women
}

\author{
K. Sathyapriya \\ Sree Saraswathi Thyagaraja \\ College
}

\begin{abstract}
In this paper a fuzzy inventory model is developed for deteriorating items with power demand rate. Shortages are allowed and partially backlogged. The holding cost is assumed to be time dependent. The cost components are considered as trapezoidal fuzzy numbers. The objective of this paper is to develop an inventory model in a fuzzy environment, minimize the total cost and thereby derive optimal policies. The total cost is defuzzified using Graded mean representation, and Signed distance methods. The values obtained by these methods are compared with the help of numerical examples. The convexity of the cost function is depicted graphically. The formulated model is tested for sensitivity by studying the effect of change in parameters.
\end{abstract}

\section{Keywords}

Defuzzification, Deterioration, Graded mean representation method, Optimization, Partial backlogging, Power Demand, Shortages, Signed Distance Method, and Trapezoidal Fuzzy Number.

\section{INTRODUCTION}

Mathematical modelling of inventory system dates back to1915 when Harris developed the first inventory model and defined the Economic Order Quantity. It was generalized by Wilson who gave a formula to obtain Economic order quantity. Whitin later formulated a model considering the deterioration of the fashion goods at the end of the prescribed shortage period. Ghare and Schrader [1] developed a significant model for an exponentially decaying inventory. Mishra [2] introduced an production inventory system with variable rate of deterioration. Later Abad [3] proposed an optimal pricing and lot-sizing policy under the conditions of perishability and partial backordering. Teng et al., [4] presented an optimal replenishment policy for deteriorating items with time-varying demand and partial backlogging. Dye et al., [5] proposed a deterministic inventory model for deteriorating items with capacity constraint and timeproportional backlogging rate. Roy et.al., [6] proposed an economic order quantity model of imperfect quality items with partial backlogging. Hui-Ling Yang [7] presented a partial backlogging inventory model for deteriorating items with fluctuating selling price and purchasing cost.

Demand being a key factor in any inventory situation, has a high impact on inventory policies. Naddor [8] analyzed various components and properties of inventory systems and pointed out that the demand process plays a significant role in the inventory management. He introduced the power demand pattern. Several research articles involving power demand have been published. Following Naddor, Goel and Aggarwal [9] developed an order-level inventory model with power demand for deteriorating items. Datta and Pal [10] studied an inventory system with power demand and variable rate of deterioration. Lee and $\mathrm{Wu}$ [11] proposed an EOQ model assuming deterioration, shortages and power demand pattern. Dye [12] extended the Lee and $\mathrm{Wu}$ model assuming a timeproportional backlogging rate. Singh et al. [13] developed an EOQ model for perishable items with power demand pattern and partial backlogging. Rajeswari and Vanjikkodi [14] developed an inventory model for deteriorating items with partial backlogging and power demand pattern. Rajeswari and Vanjikkodi [15] analyzed an inventory model for Weibull deteriorating items with power pattern demand. Mishra and Singh [16] presented an EOQ model for deteriorating items with power demand pattern and shortages partially backlogged. Recently, Sicilia et al. [17] studied a production inventory model for deteriorating items with shortages and time varying demand following a power demand pattern. They assumed a complete backlogging of orders.

When cost varies with time it is natural to expect uncertainties and thereby imprecision in defining the important parameters of the inventory problem. The conventional inventory models, deal with uncertainties using either probabilistic or stochastic concepts. But in reality, uncertainties occur due to fuzziness of the situation. Such situations can be dealt more comfortably using the fuzzy set theory proposed by Zadeh in [18]. Among the three possibilities, fuzziness is the closest possible representation to reality, and thereby it can be considered as an authentic expression of an inventory situation with some uncertainity.

Initially, Kauffmann and Gupta [19] dealt with fuzzy arithmetic operations and Zimmermann [20] discussed the concept of the fuzzy set theory and its applications. Yao and Lee [21] introduced an inventory model with fuzzy order quantity considering triangular and trapezoidal fuzzy numbers, with shortage cost as a crisp parameter. J. S. Yao and H.M. Lee [22] developed a Fuzzy Inventory with or without Backorder for Fuzzy Order Quantity with Trapezoidal Fuzzy Number. Yao et al. [23] assumed the order quantity and the total demand rate as triangular fuzzy numbers and obtained a fuzzy inventory model. Dutta et al. [24] developed a model in presence of fuzzy random variable demand where the optimum is achieved using a graded mean integration representation.

Roy and Samanta [25] developed a fuzzy continuous review inventory model without backorder for deteriorating items where cycle time is considered as a symmetric fuzzy number. They used the signed distance method to defuzzify the total cost. D. Dutta and Pawan Kumar [26] used trapezoidal fuzzy numbers in their fuzzy inventory model without shortages. Dutta, and Pavan Kumar [27] determined an optimal ordering 
policy for an inventory model with deteriorating items and shortages by considering demand rate, ordering cost and holding cost as trapezoidal fuzzy numbers. Recently, Sushil Kumar and Rajput [28] proposed fuzzy inventory model for deteriorating items with time dependent demand and partial backlogging.

The objective of this paper is to determine the optimum inventory policy for an inventory system with power demand and a time-dependent holding cost in fuzzy environment.Here deteriorating cost, holding cost, unit cost, backorder cost and lost sale cost are taken as trapezoidal fuzzy numbers. For defuzzification of the total cost function. Graded mean and Signed distance methods are used. The values obtained by these methods are compared with the help of numerical examples. The convexity of the cost function is depicted graphically. Sensitivity analysis is performed to study the effect of change of some parameters

\section{PRELIMINARIES}

\section{Definition 2.1: Fuzzy Number}

A fuzzy number is a convex normalized fuzzy set defined on $\mathrm{R}$ whose membership function is at least piecewise continuous. The membership function $\mu_{\tilde{\AA}}$ for the fuzzy number $\tilde{\mathrm{A}}$, is a mapping of the form $\mu_{\tilde{\mathrm{A}}}: \mathrm{X} \rightarrow[0,1]$, where $\mathrm{X}$ is the universal set.

Definition 2.2: A trapezoidal fuzzy number $A=(a, b, c, d)$ is represented by a membership function $\mu_{\mathrm{A}}$ as:

$$
\mu_{A}(x)= \begin{cases}0, & x<a_{1} \\ \frac{x-a_{1}}{a_{2}-a_{1}}, & a_{1} \leq x \leq a_{2} \\ 1, & a_{2} \leq x \leq a_{3} \\ \frac{a_{4}-x}{a_{4}-a_{3}}, & a_{3} \leq x \leq a_{4} \\ 0, & x>a_{4}\end{cases}
$$

Definition 2.3: Operations on Trapezoidal fuzzy numbers

Suppose $\tilde{\mathrm{A}}=\left(\mathrm{a}_{1}, \mathrm{a}_{2}, \mathrm{a}_{3}, \mathrm{a}_{4}\right)$ and $\tilde{\mathrm{B}}=\left(\mathrm{b}_{1}, \mathrm{~b}_{2}, \mathrm{~b}_{3}, \mathrm{~b}_{4}\right)$ are two trapezoidal fuzzy numbers, then arithmetical operations on these numbers are defined as:
(i) $\tilde{\mathrm{A}}+\tilde{\mathrm{B}}=\left(\mathrm{a}_{1}+\mathrm{b}_{1}, \mathrm{a}_{2}+\mathrm{b}_{2}, \mathrm{a}_{3}+\mathrm{b}_{3}, \mathrm{a}_{4}+\mathrm{b}_{4}\right)$
(ii) $\tilde{\mathrm{A}}-\tilde{\mathrm{B}}=\left(\mathrm{a}_{1}-\mathrm{b}_{4}, \mathrm{a}_{2}-\mathrm{b}_{3}, \mathrm{a}_{3}-\mathrm{b}_{2}, \mathrm{a}_{4}-\mathrm{b}_{1}\right)$
(iii) $\tilde{\mathrm{A}} \times \tilde{\mathrm{B}}=\left(\mathrm{a}_{1} \mathrm{~b}_{1}, \mathrm{a}_{2} \mathrm{~b}_{2}, \mathrm{a}_{3} \mathrm{~b}_{3}, \mathrm{a}_{4} \mathrm{~b}_{4}\right)$

Definition 2.4: The $\alpha$ - cut of $\tilde{A}=(a, b, c, d) \in F_{N}, 0 \leq \alpha \leq 1$, is defined by $\mathrm{A}(\alpha)=\left[\mathrm{A}_{\mathrm{L}}(\alpha), \mathrm{A}_{\mathrm{R}}(\alpha)\right]$.

Where $A_{L}(\alpha)=a+(b-a) \alpha$ and $A_{R}(\alpha)=d-(d-c) \alpha$ are the left and right endpoints of $\mathrm{A}(\alpha)$.

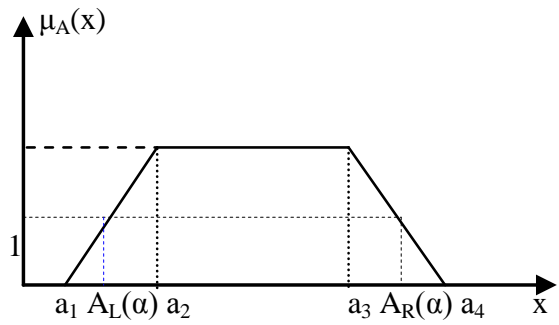

Figure 1: $\alpha$-Cut of a Trapezoidal Fuzzy Number
Definition 2.5:If $\tilde{A}=(a, b, c, d)$ is a trapezoidal fuzzy number then the graded mean integration representation of $\tilde{A}$ is defined as

$$
\begin{aligned}
& P(\tilde{A})=\frac{\int_{0}^{w_{A}} h\left(\frac{L^{-1}(h)+R^{-1}(h)}{2}\right) d h}{\int_{0}^{w_{A}} h d h} \text { with } 0<h \leq w_{A} \text { and } 0<w_{A} \leq 1 . \\
& P(\tilde{A})=(1 / 2) \frac{\int_{0}^{1} h[a+h(b-a)+d-h(d-c)] d h}{\int_{0}^{1} h d h}=\frac{a+2 b+2 c+d}{6}
\end{aligned}
$$

Definition 2.6: If $\tilde{A}=(a, b, c, d)$ is a trapezoidal fuzzy number then the signed distance of $\tilde{\mathrm{A}}$ is defined as

$$
\mathrm{d}(\tilde{\mathrm{A}}, \tilde{\mathrm{O}})=\int_{0}^{1} \mathrm{~d}\left(\left[\mathrm{~A}_{\mathrm{L}}(\alpha)_{\alpha}, \mathrm{A}_{\mathrm{R}}(\alpha)_{\alpha}\right], \tilde{\mathrm{O}}\right)=\frac{1}{4}(\mathrm{a}+\mathrm{b}+\mathrm{c}+\mathrm{d})
$$

\section{NOTATIONS AND ASSUMPTIONS 3.1 Notations}

A - The ordering cost per order.

C - The purchase cost per unit.

$h(t)-(a+b t)$ holding cost per unit per time unit.

$\pi_{\mathrm{b}}-$ The backordered cost per unit short per time unit.

$\pi_{1}-$ The cost of lost sales per unit.

$\pi_{\mathrm{d}}-$ The deteriorating cost per unit

$\theta$ - The deterioration rate

$t_{1} \quad$ The time at which the inventory level reaches zero, $\mathrm{t}_{1} \geq 0$.

$t_{2}$ - Portion of the cycle time during which shortages are allowed, $\mathrm{t}_{2} \geq 0$.

$\mathrm{T}-\left(=\mathrm{t}_{1}+\mathrm{t}_{2}\right)$ The length of cycle time.

$\mathrm{I}_{\mathrm{MI}}$ - The maximum inventory level during $[0, \mathrm{~T}]$.

$\mathrm{I}_{\mathrm{MB}}$ - The maximum backordered units during stock out period.

Q - $\left(=\mathrm{I}_{\mathrm{MI}}+\mathrm{I}_{\mathrm{MB}}\right)$ The order quantity during a cycle of length $\mathrm{T}$.

$I_{1}(t)-$ The level of positive inventory at time $t, 0 \leq t \leq t_{1}$. $I_{2}(t)-$ The level of backordered units at time $t, t_{1} \leq t \leq T$. TCUT - The total cost per time unit.

$\tilde{\mathrm{C}}-$ Fuzzy purchase cost per unit.

$\tilde{\mathrm{h}}(\mathrm{t})-(\tilde{\mathrm{a}}+\mathrm{bt})$ fuzzy holding cost per unit per time unit.

$\tilde{\pi}_{\mathrm{b}}-$ Fuzzy backordered cost per unit short per time unit.

$\tilde{\pi}_{1}-$ Fuzzy cost of lost sales per unit.

$\tilde{\pi}_{\mathrm{d}}$ - Fuzzy deteriorating cost per unit.

TCUT̃ - Fuzzy total cost per time unit

\subsection{Assumptions}

- The inventory system deals with single item.

- The demand rate $\frac{\mathrm{dt}^{(1-\mathrm{n}) / \mathrm{n}}}{n T^{1 / n}}$ at any time $\mathrm{t}$, where $\mathrm{d}$ is a positive constant, $\mathrm{n}$ may be any positive number, $\mathrm{T}$ is the planning horizon. $\mathrm{T}=1$.

- Time dependent deterioration rate is $\theta(\mathrm{t})=\theta \mathrm{t}$, $0<\theta<<1, \mathrm{t}>0$.

- Holding cost is time dependent and linear. That is, $h(t)=a+b t$. 
- The replenishment rate is infinite.

- The lead-time is zero or negligible.

- The planning horizon is infinite.

- During the stock out period, the backlogging rate is variable and is dependent on the length of the waiting time for the next replenishment. The proportion of the customers who would like to accept the backlogging at time " $t$ " is with the waiting time $(\mathrm{T}-\mathrm{t})$ for the next replenishment. That is for the negative inventory the backlogging rate is
$\mathrm{B}(\mathrm{t})=\frac{1}{1+\delta(\mathrm{T}-\mathrm{t})} ; \delta>0$ denotes the backlogging parameter and $\mathrm{t}_{1} \leq \mathrm{t} \leq \mathrm{T}$.

- Holding cost, deteriorating cost, back order cost, last sale cost and the purchase cost are fuzzifiied.

\section{MATHEMATICAL MODEL}

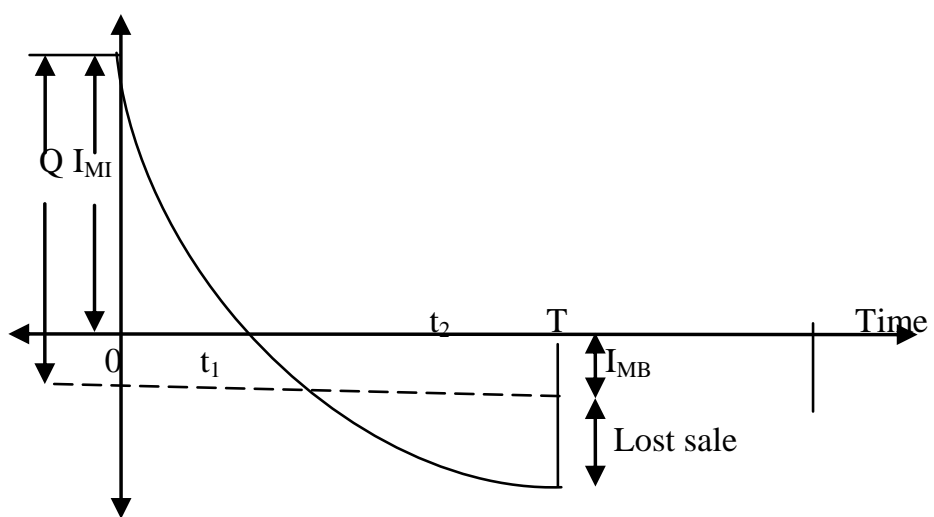

Figure 2: Representation of inventory system

\subsection{Crisp model}

\subsubsection{Inventory level before shortage period}

During the period $\left[0, \mathrm{t}_{1}\right]$, the stock level changes due to the deterioration and demand. Hence, the differential equation that governs the inventory level $\mathrm{I}_{1}(\mathrm{t})$ at any time $\mathrm{t}$ during the cycle $\left[0, \mathrm{t}_{1}\right]$ is given by

$\frac{d I_{1}(t)}{d t}+\theta t_{1}(t)=-\frac{\mathrm{dt}^{\frac{1-n}{n}}}{n^{\frac{1}{n}}}, \quad 0 \leq t \leq t_{1}$

with the condition $\mathrm{I}_{1}\left(\mathrm{t}_{1}\right)=0$ at $\mathrm{t}=\mathrm{t}_{1}$

Hence the inventory level at any instant $\mathrm{t}$ is,

$$
\begin{gathered}
\mathrm{I}_{1}(\mathrm{t})=\frac{\mathrm{d}}{\mathrm{T}^{\frac{1}{\mathrm{n}}}}\left[\left(1-\frac{\theta \mathrm{t}^{2}}{2}\right)\left(\mathrm{t}_{1}^{\frac{1}{\mathrm{n}}}-\mathrm{t}^{\frac{1}{\mathrm{n}}}\right)+\frac{\theta}{2(1+2 \mathrm{n})}\left(\mathrm{t}_{1}^{\frac{1+2 \mathrm{n}}{\mathrm{n}}}-\mathrm{t}^{\frac{1+2 \mathrm{n}}{\mathrm{n}}}\right)\right] \\
0 \leq \mathrm{t} \leq \mathrm{t}_{1}
\end{gathered}
$$

\subsubsection{Inventory level during shortage period}

During the interval $\left[\mathrm{t}_{1}, \mathrm{~T}\right]$ the inventory level depends on demand. In this period a fraction of demand is backlogged. The state of inventory during $\left[\mathrm{t}_{1}, \mathrm{~T}\right]$ can be represented by the differential equation,

$$
\frac{\mathrm{dI}_{2}(\mathrm{t})}{\mathrm{dt}}=-\frac{\left(\frac{\mathrm{dt}^{\frac{1-\mathrm{n}}{\mathrm{n}}}}{\mathrm{n} \mathrm{T}^{\frac{1}{\mathrm{n}}}}\right)}{1+\delta(\mathrm{T}-\mathrm{t})}, \quad \mathrm{t}_{1} \leq \mathrm{t} \leq \mathrm{T}
$$

with the boundary condition $\mathrm{I}_{2}\left(\mathrm{t}_{1}\right)=0$ at $\mathrm{t}=\mathrm{t}_{1}$.

The solution of equation (3) is given by

$I_{2}(t)=-\frac{d}{T^{\frac{1}{n}}}\left[(1-\delta T)\left(t^{\frac{1}{n}}-t_{1}^{\frac{1}{n}}\right)+\frac{\delta}{1+n}\left(t^{\frac{1+n}{n}}-t_{1}^{\frac{1+n}{n}}\right)\right], \quad t_{1} \leq t \leq T$
The maximum positive inventory is

$$
\mathrm{I}_{\mathrm{MI}}=\mathrm{I}_{1}(0)=\frac{\mathrm{d}}{\mathrm{T}^{\frac{1}{\mathrm{n}}}}\left[\mathrm{t}_{1}^{\frac{1}{\mathrm{n}}}+\frac{\theta \mathrm{t}^{\frac{1+2 \mathrm{n}}{{ }^{\mathrm{n}}}}}{2(1+2 \mathrm{n})}\right]
$$

The maximum backordered units are

$$
\mathrm{I}_{\mathrm{MB}}=-\mathrm{I}_{2}(\mathrm{~T})=\frac{\mathrm{d}}{\mathrm{T}^{\frac{1}{\mathrm{n}}}}\left[(1-\delta \mathrm{T})\left(\mathrm{T}^{\frac{1}{\mathrm{n}}}-\mathrm{t}_{1}^{\frac{1}{\mathrm{n}}}\right)+\frac{\delta}{1+\mathrm{n}}\left(\mathrm{T}^{\frac{1+\mathrm{n}}{\mathrm{n}}}-\mathrm{t}_{1}^{\frac{1+\mathrm{n}}{\mathrm{n}}}\right)\right]
$$

Hence, the order size during $[0, T]$ is $Q=I_{M I}+I_{M B}$.

$$
\mathrm{Q}=\frac{\mathrm{d}}{\mathrm{T}^{\frac{1}{\mathrm{n}}}}\left[\mathrm{T}^{\frac{1}{\mathrm{n}}}+\frac{\theta \mathrm{t}_{1}^{\frac{1+2 \mathrm{n}}{\mathrm{n}}}}{2(1+2 \mathrm{n})}+\delta \mathrm{Tt}_{1}^{\frac{1}{\mathrm{n}}}-\frac{\delta}{1+\mathrm{n}}\left(\mathrm{nT}^{\frac{1+\mathrm{n}}{\mathrm{n}}}+\mathrm{t}_{1}^{\frac{1+\mathrm{n}}{\mathrm{n}}}\right)\right]
$$

\subsubsection{Cost components:}

The total cost per replenishment cycle consists of the following cost components.

\section{Ordering cost per cycle}

$\mathrm{I}_{\mathrm{OC}}=\mathrm{A}$

Inventory holding cost per cycle

$I_{H C}=h \int_{0}^{t_{1}} I_{1}(t) d t=\frac{d}{T^{\frac{1}{n}}}\left[\frac{a t_{1}^{\frac{1+n}{n}}}{1+n}+\frac{a \theta t_{1}^{\frac{1+3 n}{n}}}{3(1+3 n)}+\frac{b t_{1}^{\frac{1+2 n}{n}}}{1+2 n}+\frac{b \theta t_{1}^{\frac{1+4 n}{n}}}{8(1+4 n)}\right]$

Backordered cost per cycle

$I_{B C}=\pi_{b} \int_{t_{1}}^{T}\left(-I_{2}(t)\right) d t=\frac{\pi_{b} d}{T^{\frac{1}{n}}}\left[\left(\delta T^{2}-T\right) t_{1}^{\frac{1}{n}}+\frac{n T^{\frac{1+n}{n}}}{1+n}+\frac{(1-2 \delta T) t_{1}^{\frac{1+n}{n}}}{1+n}\right.$ 


$$
\left.-\frac{2 \delta n^{2} T^{\frac{1+2 n}{n}}}{(1+n)(1+2 n)}+\frac{\delta t_{1}^{\frac{1+2 n}{n}}}{1+2 n}\right]
$$

\section{Cost due to lost sales per cycle}

$$
\mathrm{I}_{\mathrm{LS}}=\pi_{1} \int_{\mathrm{t}_{1}}^{\mathrm{T}}\left[1-\frac{1}{1+\delta(\mathrm{T}-\mathrm{t})}\right]\left(\frac{\mathrm{dt}^{\frac{1-\mathrm{n}}{\mathrm{n}}}}{\mathrm{nT}^{\frac{1}{\mathrm{n}}}}\right) \mathrm{dt}=\frac{\pi_{1} \mathrm{~d} \delta}{\mathrm{T}^{\frac{1}{\mathrm{n}}}}\left[\frac{\mathrm{nT}^{\frac{1+\mathrm{n}}{\mathrm{n}}}}{1+\mathrm{n}}-\mathrm{Tt}_{1}^{\frac{1}{\mathrm{n}}}+\frac{\mathrm{t}_{1}^{\frac{1+\mathrm{n}}{n}}}{1+\mathrm{n}}\right]
$$

\section{Deterioration Cost}

$$
\begin{aligned}
\mathrm{I}_{\mathrm{DC}} & =\pi_{\mathrm{d}}\left\{\mathrm{Q}-\int_{0}^{\mathrm{t}_{1}}\left(\frac{\mathrm{dt}^{\frac{(1-\mathrm{n})}{\mathrm{n}}}}{\mathrm{nT}^{\frac{1}{\mathrm{n}}}}\right) \mathrm{dt}-\int_{\mathrm{t}_{1}}^{\mathrm{T}} \frac{1}{1+\delta(\mathrm{T}-\mathrm{t})}\left(\frac{\mathrm{dt}^{\frac{1-\mathrm{n}}{\mathrm{n}}}}{\mathrm{nT}^{\frac{1}{\mathrm{n}}}}\right) \mathrm{dt}\right\} \\
& =\pi_{\mathrm{d}} \frac{\mathrm{d} \theta \mathrm{t}_{1}^{\frac{1+2 \mathrm{n}}{\mathrm{n}}}}{2(1+2 \mathrm{n}) \mathrm{T}^{\frac{1}{\mathrm{n}}}}
\end{aligned}
$$

\section{Purchase cost per cycle}

$\mathrm{I}_{\mathrm{PC}}=\mathrm{C} \times \mathrm{Q}=\frac{\mathrm{Cd}}{\mathrm{T}^{\frac{1}{\mathrm{n}}}}\left[\mathrm{T}^{\frac{1}{\mathrm{n}}}+\frac{\theta \mathrm{t}_{1}^{\frac{1+2 \mathrm{n}}{\mathrm{n}}}}{2(1+2 \mathrm{n})}+\delta \mathrm{Tt}_{1}^{\frac{1}{\mathrm{n}}}-\frac{\delta}{1+\mathrm{n}}\left(\mathrm{nT}^{\frac{1+\mathrm{n}}{\mathrm{n}}}+\mathrm{t}_{1}^{\frac{1+\mathrm{n}}{\mathrm{n}}}\right)\right]$

Hence, the total cost per time unit is

$$
\begin{aligned}
& \text { TCUT }=\frac{1}{\mathrm{~T}}\left[\mathrm{I}_{\mathrm{OC}}+\mathrm{I}_{\mathrm{HC}}+\mathrm{I}_{\mathrm{BC}}+\mathrm{I}_{\mathrm{LS}}+\mathrm{I}_{\mathrm{PC}}\right] \\
& \text { TCUT }=\frac{1}{\mathrm{~T}}\left\{\mathrm{~A}+\frac{\pi_{\mathrm{b}} \mathrm{d}}{\mathrm{T}^{\frac{1}{\mathrm{n}}}}\left[\left(\delta \mathrm{T}^{2}-\mathrm{T}\right) \mathrm{t}_{1}^{\frac{1}{\mathrm{n}}}+\frac{\mathrm{nT} \mathrm{T}^{\frac{1+\mathrm{n}}{\mathrm{n}}}}{1+\mathrm{n}}+\frac{(1-2 \delta \mathrm{T}) \mathrm{t}_{1}^{\frac{1+\mathrm{n}}{\mathrm{n}}}}{1+\mathrm{n}}\right.\right. \\
& \left.-\frac{2 \delta n^{2} T^{\frac{1+2 n}{n}}}{(1+n)(1+2 n)}+\frac{\delta t_{1}^{\frac{1+2 n}{n}}}{1+2 n}\right]+\frac{\pi_{1} d \delta}{T^{\frac{1}{n}}}\left[\frac{n T^{\frac{1+n}{n}}}{1+n}-T_{1}^{\frac{1}{n}}+\frac{t_{1}^{\frac{1+n}{n}}}{1+n}\right] \\
& +\frac{\pi_{d} d \theta t_{1}^{\frac{1+2 n}{n}}}{2(1+2 n) T^{\frac{1}{n}}}+\left[\frac{a t_{1}^{\frac{1+n}{n}}}{1+n}+\frac{a \theta t_{1}^{\frac{1+3 n}{n}}}{3(1+3 n)}+\frac{b t_{1}^{\frac{1+2 n}{n}}}{1+2 n}+\frac{b \theta t_{1}^{\frac{1+4 n}{n}}}{8(1+4 n)}\right] \\
& \left.+\frac{\mathrm{Cd}}{\mathrm{T}^{\frac{1}{\mathrm{n}}}}\left[\mathrm{T}^{\frac{1}{\mathrm{n}}}+\frac{\theta \mathrm{t}_{1}^{\frac{1+2 \mathrm{n}}{\mathrm{n}}}}{2(1+2 \mathrm{n})}+\delta \mathrm{Tt}_{1}^{\frac{1}{\mathrm{n}}}-\frac{\delta}{1+\mathrm{n}}\left(\mathrm{nT}^{\frac{1+\mathrm{n}}{\mathrm{n}}}+\mathrm{t}_{1}^{\frac{1+\mathrm{n}}{\mathrm{n}}}\right)\right]\right\}
\end{aligned}
$$

The optimal cycle time is obtained by solving the equation

$$
\frac{\mathrm{dTCUT}}{\mathrm{dt}_{1}}=0
$$

The Equation (15) is nonlinear. It is solved using MATLAB 7. The minimum total average cost per unit time (TCUT), and Q can be obtained by using the value of $t_{1}$ in (14) and (7).

The sufficiency condition for a local minimum is also verified for the value of $t_{1}$ obtained from (15).

\subsection{Fuzzy Model}

Fuzzy values (trapezoidal fuzzy numbers.) for the parameters namely $\tilde{\mathrm{C}}, \tilde{\pi}_{\mathrm{b}}, \tilde{\mathrm{a}}, \tilde{\pi}_{1}, \tilde{\pi}_{\mathrm{d}}$ are taken as:

$$
\begin{aligned}
& \tilde{\pi}_{1}=\left(\pi_{11}, \pi_{12}, \pi_{13}, \pi_{14}\right), \tilde{\pi}_{b}=\left(\pi_{\mathrm{b} 1}, \pi_{\mathrm{b} 2}, \pi_{\mathrm{b} 3}, \pi_{\mathrm{b} 4}\right), \\
& \tilde{\mathrm{C}}=\left(\mathrm{C}_{1}, \mathrm{C}_{2}, \mathrm{C}_{3}, \mathrm{C}_{4}\right), \tilde{\mathrm{a}}=\left(\mathrm{a}_{1}, \mathrm{a}_{2}, \mathrm{a}_{3}, \mathrm{a}_{4}\right), \\
& \tilde{\pi}_{\mathrm{d}}=\left(\pi_{\mathrm{d} 1}, \pi_{\mathrm{d} 2}, \pi_{\mathrm{d} 3}, \pi_{\mathrm{d} 4}\right)
\end{aligned}
$$

Total cost of the inventory per unit time in fuzzy sense is given by

$\operatorname{TCUT}=\frac{1}{\mathrm{~T}}\left\{\mathrm{~A}+\frac{\tilde{\pi}_{1} \mathrm{~d} \delta}{\mathrm{T}^{\frac{1}{\mathrm{n}}}}\left[\frac{\mathrm{nT}^{\frac{1+\mathrm{n}}{\mathrm{n}}}}{1+\mathrm{n}}-\mathrm{Tt}_{1}^{\frac{1}{\mathrm{n}}}+\frac{\mathrm{t}_{1}^{\frac{1+\mathrm{n}}{\mathrm{n}}}}{1+\mathrm{n}}\right]\right.$

$$
\begin{aligned}
& +\frac{\tilde{\pi}_{b} d}{T^{\frac{1}{n}}}\left[\left(\delta T^{2}-T\right) t_{1}^{\frac{1}{n}}+\frac{n T^{\frac{1+n}{n}}}{1+n}+\frac{(1-2 \delta T) t_{1}^{\frac{1+n}{n}}}{1+n}\right. \\
& \left.-\frac{2 \delta n^{2} T^{\frac{1+2 n}{n}}}{(1+n)(1+2 n)}+\frac{\delta t_{1}^{\frac{1+2 n}{n}}}{1+2 n}\right]+\frac{\tilde{\pi}_{d} d \theta t_{1}^{\frac{1+2 n}{n}}}{2(1+2 n) T^{\frac{1}{n}}} \\
& +\left[\frac{\tilde{a}_{1}^{\frac{1+n}{n}}}{1+n}+\frac{\tilde{a} \theta t_{1}^{\frac{1+3 n}{n}}}{3(1+3 n)}+\frac{b_{1}^{\frac{1+2 n}{n}}}{1+2 n}+\frac{b \theta t_{1}^{\frac{1+4 n}{n}}}{8(1+4 n)}\right] \\
& \left.+\frac{\tilde{\mathrm{C}} \mathrm{d}}{\mathrm{T}^{\frac{1}{\mathrm{n}}}}\left[\mathrm{T}^{\frac{1}{\mathrm{n}}}+\frac{\theta \mathrm{t}^{\frac{1+2 \mathrm{n}}{\mathrm{n}}}}{2(1+2 \mathrm{n})}+\delta \mathrm{Tt}_{1}^{\frac{1}{\mathrm{n}}}-\frac{\delta}{1+\mathrm{n}}\left(\mathrm{nT}^{\frac{1+\mathrm{n}}{\mathrm{n}}}+\mathrm{t}_{1}{ }^{\frac{1+\mathrm{n}}{\mathrm{n}}}\right)\right]\right\}
\end{aligned}
$$

The fuzzy total cost TCUT $\left(\mathrm{t}_{1}, \mathrm{~T}\right)$, is defuzzified by Graded mean representation and Signed distance .

\subsubsection{Graded Mean Representation Method,}

Total cost is given by

$\operatorname{TCUT}_{\mathrm{dG}}=\left[\mathrm{TCUT}_{\mathrm{dG}_{1}}, \mathrm{TCUT}_{\mathrm{dG}_{2}}, \mathrm{TCUT}_{\mathrm{dG}_{3}}, \mathrm{TCUT}_{\mathrm{dG}_{4}}\right]$

Where

$$
\begin{aligned}
& \operatorname{TCUT}_{\mathrm{dG}_{\mathrm{i}}}=\frac{1}{\mathrm{~T}}\left\{\mathrm{~A}+\frac{\pi_{\mathrm{li}} \mathrm{d} \delta}{\mathrm{T}^{\frac{1}{\mathrm{n}}}}\left[\frac{\mathrm{nT}^{\frac{1+\mathrm{n}}{\mathrm{n}}}}{1+\mathrm{n}}-\mathrm{Tt}_{1}^{\frac{1}{\mathrm{n}}}+\frac{\mathrm{t}_{1}^{\frac{1+\mathrm{n}}{\mathrm{n}}}}{1+\mathrm{n}}\right]\right. \\
& +\frac{\pi_{\mathrm{bi}} \mathrm{d}}{\mathrm{T}^{\frac{1}{\mathrm{n}}}}\left[\left(\delta \mathrm{T}^{2}-\mathrm{T}\right) \mathrm{t}_{1}^{\frac{1}{\mathrm{n}}}+\frac{\mathrm{nT} \mathrm{T}^{\frac{1+\mathrm{n}}{\mathrm{n}}}}{1+\mathrm{n}}+\frac{(1-2 \delta \mathrm{T}) \mathrm{t}_{1}^{\frac{1+\mathrm{n}}{\mathrm{n}}}}{1+\mathrm{n}}\right. \\
& \left.-\frac{2 \delta n^{2} T^{\frac{1+2 n}{n}}}{(1+n)(1+2 n)}+\frac{\delta t_{1}^{\frac{1+2 n}{n}}}{1+2 n}\right]+\frac{\pi_{d i} d \theta t_{1}^{\frac{1+2 n}{n}}}{2(1+2 n) T^{\frac{1}{n}}}
\end{aligned}
$$$$
\begin{aligned}
& +\left[\frac{\mathrm{a}_{\mathrm{i}} \mathrm{t}_{1}^{\frac{1+\mathrm{n}}{\mathrm{n}}}}{1+\mathrm{n}}+\frac{\mathrm{a}_{\mathrm{i}} \theta \mathrm{t}_{1}^{\frac{1+3 n}{n}}}{3(1+3 \mathrm{n})}+\frac{\mathrm{bt}_{1}^{\frac{1+2 n}{n}}}{1+2 \mathrm{n}}+\frac{\mathrm{b} \theta \mathrm{t}_{1}^{\frac{1+4 n}{n}}}{8(1+4 \mathrm{n})}\right] \\
& \left.+\frac{\mathrm{C}_{\mathrm{i}} \mathrm{d}}{\mathrm{T}^{\frac{1}{\mathrm{n}}}}\left[\mathrm{T}^{\frac{1}{\mathrm{n}}}+\frac{\theta \mathrm{t}_{1}^{\frac{1+2 n}{n}}}{2(1+2 \mathrm{n})}+\delta \mathrm{Tt}_{1}^{\frac{1}{n}}-\frac{\delta}{1+\mathrm{n}}\left(\mathrm{nT}^{\frac{1+\mathrm{n}}{\mathrm{n}}}+\mathrm{t}_{1}^{\frac{1+\mathrm{n}}{n^{n}}}\right)\right]\right\}
\end{aligned}
$$

where $\mathrm{i}=1,2,3,4$

$\mathrm{TCUT}_{\mathrm{dG}}=\frac{1}{6}\left[\mathrm{TCUT}_{\mathrm{dG}_{1}}+2 \mathrm{TCUT}_{\mathrm{dG}_{2}}+2 \mathrm{TCUT}_{\mathrm{dG}_{3}}+\mathrm{TCUT}_{\mathrm{dG}_{4}}\right]$ 
To minimize total cost function per unit time $\mathrm{TCUT}_{\mathrm{dG}}$, the optimal value of $t_{1}$ is determined from the following equation:

$$
\frac{\mathrm{dTCUT}_{\mathrm{dG}}}{\mathrm{dt}_{1}}=0
$$

Further, for the total cost function $\mathrm{TCUT}_{\mathrm{dG}}$ to be convex, the sufficiency conditions must be satisfied

That is $\frac{\mathrm{d}^{2} \mathrm{TCUT}_{\mathrm{dG}}}{\mathrm{dt}_{1}{ }^{2}}>0$

\subsubsection{Total cost - Signed Distance Method}

$\mathrm{TCUT}_{\mathrm{ds}}=\left[\mathrm{TCUT}_{\mathrm{ds}_{1}}, \mathrm{TCUT}_{\mathrm{ds}_{2}}, \mathrm{TCUT}_{\mathrm{ds}_{3}}, \mathrm{TCUT}_{\mathrm{ds}_{4}}\right]$

where $\mathrm{TCUT}_{\mathrm{dS}_{\mathrm{i}}}$ are defined by (17).

$$
\mathrm{TCUT}_{\mathrm{ds}}=\frac{1}{4}\left[\mathrm{TCUT}_{\mathrm{ds}_{1}}+2 \mathrm{TCUT}_{\mathrm{ds}_{2}}+\mathrm{TCUT}_{\mathrm{ds}_{3}}+\mathrm{TCUT}_{\mathrm{ds}_{4}}\right]
$$

The total cost function TCUT $_{\mathrm{dS}}$ has been minimized following the same process as has been stated previously. The optimal value of $t_{1}$ can be obtained by solving the equation:

$$
\frac{\mathrm{dTCUT}_{\mathrm{dS}}}{\mathrm{dt}_{1}}=0
$$

Further, for the total cost function $\mathrm{TCUT}_{\mathrm{dS}}$ to be convex.

$$
\frac{\mathrm{d}^{2} \mathrm{TCUT}_{\mathrm{ds}}}{\mathrm{dt}_{1}^{2}}>0
$$

\section{NUMERICAL EXAMPLE}

In this section the optimal value $\left(\mathrm{t}_{1}{ }^{*}\right)$, the optimal order quantity $\left(\mathrm{Q}^{*}\right)$ and the minimum total average cost $\left(\mathrm{TC}^{*}\right)$ are computed for both crisp and fuzzy model.

Consider an inventory system with following parametric values

\subsection{Crisp Model}

$\mathrm{D}=$ 50units, $\mathrm{T}=1, \mathrm{n}=2$ units, $\mathrm{A}=\$ 250$ /order, $\mathrm{C}=\$ 8$ /unit, $\mathrm{a}=0.50, \mathrm{~b}=0.05, \pi_{\mathrm{b}}=\$ 12 /$ unit/year, $\pi_{\mathrm{l}}=\$ 15 /$ unit, $\pi_{\mathrm{d}}=\$ 11 /$ unit, $\delta=0.5$ units, $\theta=0.05$.

The solution of crisp model is

TCUT $=\$ 662.8416 \cong \$ 663, \mathrm{t}_{1}=0.9380$ years, $\mathrm{Q}=50.1885$ units $\cong 50$ units.

\subsection{Fuzzy Model}

$\tilde{\mathrm{C}}=(6,7,9,11), \tilde{\mathrm{a}}=(0.3,0.4,0.6,0.8), \tilde{\pi}_{\mathrm{b}}=(10,11,13,15)$,

$\tilde{\pi}_{1}=(13,14,16,18), \quad \tilde{\pi}_{\mathrm{d}}=(9,10,12,14)$.

Optimal values corresponding to different combinations of cost parameters of the fuzzy model for different defuzzification methods is tabulated below.

Table 1 Computations with fuzzy parameters

\begin{tabular}{|c|c|c|c|c|}
\hline $\begin{array}{c}\text { Deffuzzification } \\
\text { methods }\end{array}$ & $\begin{array}{c}\text { Fuzzy } \\
\text { parameters }\end{array}$ & $\mathbf{t}_{\mathbf{1}}$ (years) & $\begin{array}{l}\text { TCUT }_{\text {Ds }} \\
\left(\mathbf{t}_{\mathbf{1}}, \mathbf{T}\right)(\$)\end{array}$ & $\mathbf{Q}$ (units) \\
\hline \multirow{4}{*}{$\begin{array}{c}\text { Graded } \\
\text { Mean } \\
\text { Representation } \\
\text { method }\end{array}$} & $\tilde{\mathrm{C}}, \tilde{\mathrm{a}}, \tilde{\pi}_{\mathrm{b}}, \tilde{\pi}_{\mathrm{l}}, \tilde{\pi}_{\mathrm{d}}$ & 0.9372 & 671.5077 & 50.1874 \\
\cline { 2 - 5 } & $\tilde{\mathrm{a}}, \tilde{\pi}_{\mathrm{b}}, \tilde{\pi}_{1}, \tilde{\pi}_{\mathrm{d}}$ & 0.9377 & 663.1431 & 50.1881 \\
\cline { 2 - 5 } & $\tilde{\pi}_{\mathrm{b}}, \tilde{\pi}_{1}, \tilde{\pi}_{\mathrm{d}}$ & 0.9387 & 662.8891 & 50.1894 \\
\cline { 2 - 5 } & $\tilde{\pi}_{\mathrm{b}}, \tilde{\pi}_{\mathrm{d}}$ & 0.9384 & 662.8851 & 50.1890 \\
\cline { 2 - 6 } & $\tilde{\pi}_{\mathrm{b}}$ & 0.9386 & 662.8495 & 50.1893 \\
\hline
\end{tabular}

\begin{tabular}{|c|c|c|c|c|}
\hline & $\tilde{\mathrm{C}}, \tilde{\mathrm{a}}, \tilde{\pi}_{\mathrm{b}}, \tilde{\pi}_{\mathrm{l}}, \tilde{\pi}_{\mathrm{d}}$ & 0.9368 & 675.8407 & 50.1868 \\
\cline { 2 - 5 } & $\tilde{\mathrm{a}}, \tilde{\pi}_{\mathrm{b}}, \tilde{\pi}_{1}, \tilde{\pi}_{\mathrm{d}}$ & 0.9376 & 663.2938 & 50.1879 \\
\cline { 2 - 5 } $\begin{array}{c}\text { Signed } \\
\text { Distance } \\
\text { Method }\end{array}$ & $\tilde{\pi}_{\mathrm{b}}, \tilde{\pi}_{1}, \tilde{\pi}_{\mathrm{d}}$ & 0.9390 & 662.9213 & 50.1899 \\
\cline { 2 - 5 } & $\tilde{\pi}_{\mathrm{b}}, \tilde{\pi}_{\mathrm{d}}$ & 0.9386 & 662.9068 & 50.1893 \\
\cline { 2 - 5 } & $\tilde{\pi}_{\mathrm{b}}$ & 0.9389 & 662.8534 & 50.1897 \\
\hline
\end{tabular}

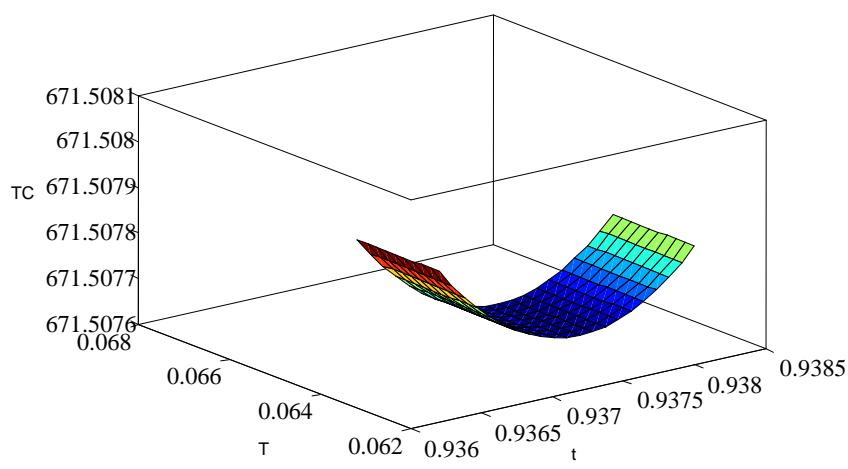

Figure 3: Total Fuzzy Cost TCUT $_{d G}\left(t_{1}, T\right)$ Vs. $t_{1}$ and $t_{2}$

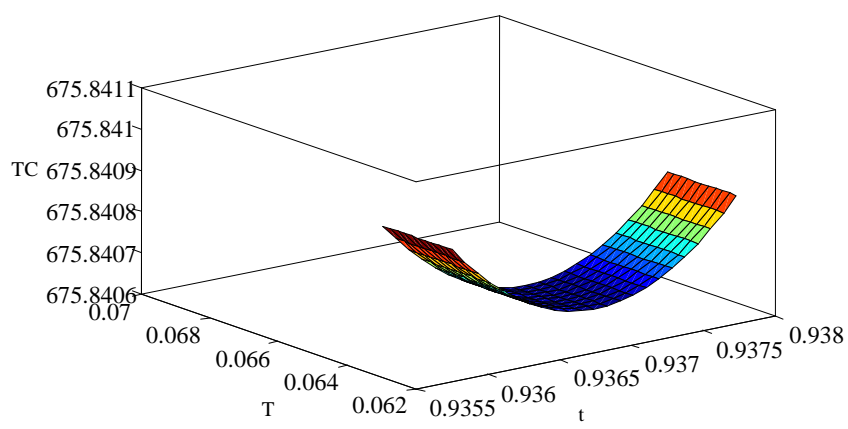

Figure 4: Total Fuzzy Cost $\operatorname{TCUT}_{\mathrm{dS}}\left(\mathrm{t}_{\mathbf{1}}, \mathrm{T}\right)$ Vs. $\mathrm{t}_{1}$ and $\mathrm{t}_{\mathbf{2}}$

The convexity of the total cost function is also verified:

$$
\begin{aligned}
& \frac{\mathrm{d}^{2} \mathrm{TCUT}}{\mathrm{dt}_{1}{ }^{2}}=419.8195>0 \frac{\mathrm{d}^{2} \mathrm{TCUT}_{\mathrm{dG}}}{\mathrm{dt}_{1}{ }^{2}}=424.6140>0 \\
& \frac{\mathrm{d}^{2} \mathrm{TCUT}_{\mathrm{dS}}}{\mathrm{dt}_{1}{ }^{2}}=427.0115>0
\end{aligned}
$$

Sensitivity Analysis:

Effect of backlogging parameter $(\delta)$ :

Varying the backlogging parameter between 0.25 to 0.75 the following table is obtained.

Table 2 Variation in backlogging parameter ' $\delta$ '

\begin{tabular}{|c|c|c|c|}
\hline $\boldsymbol{\delta}$ & $\mathbf{t}_{\mathbf{1}}(\mathbf{\text { years}})$ & $\mathbf{T C U T}_{\mathbf{d G}}\left(\mathbf{t}_{\mathbf{1}}, \mathbf{T}\right)(\mathbf{\$})$ & $\mathbf{Q}$ (units) \\
\hline $\mathbf{0 . 2 5 0}$ & 0.9301 & 671.4178 & 50.1929 \\
\hline $\mathbf{0 . 3 7 5}$ & 0.9341 & 671.4649 & 50.1900 \\
\hline $\mathbf{0 . 5 0 0}$ & 0.9372 & 671.5077 & 50.1874 \\
\hline $\mathbf{0 . 6 2 5}$ & 0.9400 & 671.5468 & 50.1855 \\
\hline $\mathbf{0 . 7 5 0}$ & 0.9427 & 671.5825 & 50.1843 \\
\hline
\end{tabular}


In the above table it can be observed that, increase in backlogging parameter

- Increases total cost per time unit of the inventory system and inventory time period.

- Decrease ordering quantity.

Effect of deterioration parameter $(\theta)$ :

Initially the deterioration parameter has been taken as 0.05 . The following table is obtained with the variation in deterioration parameter.

Table 3 Variation in deterioration parameter ' $\theta$ '

\begin{tabular}{|c|c|c|c|}
\hline $\boldsymbol{\theta}$ & $\mathbf{t}_{\mathbf{1}}(\mathbf{y e a r s})$ & $\mathbf{T C U T}_{\mathbf{d G}}\left(\mathbf{t}_{\mathbf{1}} \mathbf{1}, \mathbf{T}\right)(\mathbf{\$})$ & $\mathbf{Q}$ (units) \\
\hline $\mathbf{0 . 0 2 5 0}$ & 0.9506 & 669.3902 & 50.0946 \\
\hline $\mathbf{0 . 0 3 7 5}$ & 0.9438 & 670.4584 & 50.1421 \\
\hline $\mathbf{0 . 0 5 0 0}$ & 0.9372 & 671.5077 & 50.1874 \\
\hline $\mathbf{0 . 0 6 2 5}$ & 0.9307 & 672.5387 & 50.2304 \\
\hline $\mathbf{0 . 0 7 5 0}$ & 0.9244 & 673.5520 & 50.2714 \\
\hline
\end{tabular}

In the above table it can be observed that, increase in deteriorating parameter

- Increases total cost per time unit of an inventory system and ordering quantity.

- Decrease in inventory time period.

\section{CONCLUSION}

A fuzzy inventory model for deteriorating items with allowable shortages and power demand has been developed. Cost parameters are represented by trapezoidal fuzzy numbers. For defuzzification, graded mean and signed distance methods are used to evaluate the optimal time period of positive stock and order quantity in which minimizes the total cost. Numerical examples are used to validate the model. It is concluded that graded mean representation method gives minimum cost as compared to Signed distance method. Sensitivity analysis is also conducted on the parameters to explore the effects of fuzziness.

\section{REFERENCES}

[1] Ghare P.M, Schader GF 1963, A model for an exponentially decaying inventory. J Ind Engineering 14, 238-243.

[2] Misra, R.B 1975, Optimum production lot size model for a system with deteriorating inventory. I.J.P.E., 13, 495505 .

[3] Abad PL 1996, optimal pricing and lot-sizing under conditions of perishability and partial backordering. Manage Sci 42, 1093-1104.

[4] J.-T. Teng, H.-J. Chang, C.-Y. Dye, and C.-H. Hung 2002, An optimal replenishment policy for deteriorating items with time-varying demand and partial backlogging, Operations Research Letters, 30(6), 387-393.

[5] Dye C.Y., Ouyang L.Y. and Hsieh T.P. 2007, Deterministic inventory model for deteriorating items with capacity constraint and time-proportional backlogging rate. Eur J Oper Res., 178(3),789-807.

[6] M. Das Roy, S.S. Sana and K. Chaudhuri 2011, An economic order quantity model of imperfect quality items with partial backlogging, International Journal of Systems Science. Principles and Applications of Systems and Integration, 42(8), 1409-1419.
[7] Hui-Ling Yang 2012, A partial backlogging inventory model for deteriorating items with fluctuating selling price and purchasing cost. Hindawi Publishing Corporation, Advances in Operations Research Volume 2012, Article ID 385371, 15 pages doi:10.1155/2012/385371

[8] E. Naddor 1966, Inventory Systems. John Wiley and Sons, New York.

[9] V.P. Goel and S.P. Aggarwal 1981, Order level inventory system with power demand pattern for deteriorating items. In: Proceedings of the All Indian Seminar on Operational Research and Decision Making, University of Delhi, New Delhi, 19-34.

[10] T.K. Datta and A.K. Pal 1988, Order level inventory system with power demand pattern for items with variable rate of deterioration, Indian J. Pure Appl. Math. 19(11), 1043-1053.

[11] W.C. Lee, J.W. Wu 2002, An EOQ model for items with Weibull distributed deterioration, shortages and power demand pattern. Int. J. Inf. Manag. Sci., 13(2), 19-34.

[12] Dye ,C.Y., 2004, A note on "An EOQ model for items with Weibull distributed deterioration, shortages and power demand pattern”. Int. J. Inf. Manag. Sci., 15(2), $81-84$

[13] Singh, T.J., Singh ,S.R. and Dutt ,R., 2009, An EOQ model for perishable items with power demand and partial back ordering. Int. J. Op. Quant. Manag., 15(1), $65-72$.

[14] Rajeswari.N and Vanjikkodi.T 2011, Deteriorating inventory model with power demand and partial back logging, Int. J. Math. Arch., 2(9),1495-1501.

[15] Rajeswari.N and Vanjikkodi.T 2012, An Inventory Model for Items with Two Parameter Weibull Distribution Deterioration and Backlogging. American Journal of Operations Research, 2, 247-252.

[16] Mishra .S and Singh .P.K 2013, Partial back logging EOQ model for queued customers with power demand and quadratic deterioration: computational approach. Am. J. Op. Res., 3(2), 13-27.

[17] Sicilia, J., et al., 2014, An inventory model for deteriorating items with shortages and time-varying demand. International Journal of Production Economics, $155,155-162$

[18] L.A. Zadeh 1965, Fuzzy sets, Information Control, 8, 338-353.

[19] Kaufmann .A and Gupta .M.M, 1991, Introduction to Fuzzy Arithmetic: Theory and Applications, Van Nostrand Reinhold, New York.

[20] Zimmermann .H.J, 1996, Fuzzy Set Theory and Its Applications, 3rd Ed. Dordrecht: Kluwer, Academic Publishers.

[21] Yao .J.S and Lee .H.M, 1996. Fuzzy inventory with backorder for fuzzy order quantity, Information Sciences, vol.93, pp. 283-319. 
[22] Yao, J.S. and Lee, H.M. 1999. Fuzzy Inventory with or without Backorder for Fuzzy Order Quantity with Trapezoidal Fuzzy Number. Fuzzy Sets and Systems, $105,311-337$.

[23] Yao .J.S, Chang .S.C and Su .J.S, 2000, Fuzzy Inventory without backorder for fuzzy order quantity and fuzzy total demand quantity, Computer and Operations Research, 27, 935-962.

[24] Dutta .P, Dabjani Chakraborty and Akhil Roy 2005, A single-period inventory model with fuzzy random variable demand, Mathematical and Computer Modelling, 41(8-9), 915-922.

[25] Ajanta Roy and Samanta .G.P 2009, Fuzzy continuous review inventory model without backorder for deteriorating items, Electronic Journal of Applied Statistical Analysis, 2(1), 58-66.

[26] Dutta. D and Kumar. P 2012, Fuzzy Inventory Model without Shortage Using Trapezoidal Fuzzy Number with Sensitivity Analysis. IOSR Journal of Mathematics, 4, 32-37.

[27] Dutta. D and Kumar. P 2013, Fuzzy Inventory Model for Deteriorating Items with Shortages under Fully Backlogged Condition. International Journal of Soft Computing and Engineering, 3(2).

[28] Sushil Kumar and Rajput.U.S, 2015, Fuzzy Inventory Model for Deteriorating Items with Time Dependent Demand and Partial Backlogging. Applied Mathematics, $6,496-509$. 